\title{
Households Health Status in Saudi Arabia: Spatial Distribution and Association With Socioeconomic Factors
}

\author{
Saleh Alnahdi ${ }^{1}$ \\ ${ }^{1}$ Department of Health Service and Hospitals Management, College of Business, University of Jeddah, Jeddah City, \\ Kingdom of Saudi Arabia \\ Correspondence: Saleh Alnahdi, Department of Health Service and Hospitals Management, College of Business, \\ University of Jeddah, Jeddah City, Kingdom of Saudi Arabia. E-mail: Saaalnahdi@uj.edu.sa
}

Received: May 10, 2020

doi:10.5430/rwe.v11n3p284
Accepted: June 19, 2020

Online Published: June 27, 2020

URL: https://doi.org/10.5430/rwe.v11n3p284

\begin{abstract}
This paper examines the spatial relationship between Saudi and non-Saudi people's health status and the socioeconomic composition of the neighbourhoods in which they live. Data were recorded from the National Population Health Survey (NPHS) performed by the Saudi General Authority for Statistics (GAS) in 2018. The survey counts 23,980,846 inhabitants grouped into 24,012 households who assessed their health status by gender and administrative region. Only people who are fifteen years of age and over and claiming poor health status were retained in the analysis. We used a Generalized Linear Spatial Model (GLSM) to study the relationship between Saudi and non-Saudi household's health status and socioeconomic factors. A Gaussian process with a powered exponential spatial correlation function was introduced on the right-hand side of the model to consider the unexplained spatial variation in the data. The statistical results show the progressive increase in the number of Saudi and non-Saudi households claiming poor health status with the high Saudi unemployment rate, low average monthly income and high current daily smokers. The results of the statistical analyses show the wider potential of GLSM for analyzing data of this kind and the important risk of misleading interpretations when the non-spatial analysis is used on spatially structured data. The method of inference was Bayesian using Markov Chain Monte Carlo Implementation.
\end{abstract}

Keywords: health status, households, spatial distribution, socioeconomic factors, generalized linear spatial models, Saudi Arabia

\section{Introduction}

The Kingdom of Saudi Arabia lies in the furthermost part of southwestern Asia. It has been divided into (13) administrative regions. Each one of them is divided into several governorates differentiating in number from one region to another. Saudi Arabia's population is 32.552,336, living in 3,591,098 households with a higher concentration in Al-Riyadh, Makkah Al-Mokarramah, and the Eastern Region. Good health is a key to the well-being and quality of life of the citizens as well as to economic growth and sustainable development. Thus, good knowledge and understanding of Saudi and non-Saudi household's health status are a requisite for building human capital and full participation.

In Saudi Arabia, the health of the population is defined as a special social interest. Both the Saudi government and private sector have made extensive efforts to establish an efficient healthcare system, through better decisions to improve their healthcare programs. However, improve the health status of such population, can only be achieved through a better understanding of the ways in which social, cultural, environmental, occupational, and economic factors determine health status.

In a review of the literature dealing with Saudi population health, several comparatives analyses on European and OECD countries have shown that use of health care is strongly depended on socioeconomic status of persons. Those in a more favourable socioeconomic position are more likely to access and use services (Van et al. (2006); Regidor et al. (2008); Bago et al. (2009); Devaux et al. (2008)). Namely, Hilary et al. (2018) used a descriptive analysis to examine the relationship between social factors and a person's overall health and health outcomes. They found that it is important to integrate of social determinants into the health care system. Jenni and Lauri (2020) used descriptive methods and multinomial logistic regression models to show how the probability of using health care organized by 
public, occupational and private schemes differed by socioeconomic status in a working-age population. The used covariates were socioeconomics factors linked for the total population aged 25-64 of the city of Oulu, Finland. The results reflect the design of the Finnish health care system, with a strong occupational health care scheme for the employed population contributing to inequality in use of health care and potentially to health inequality between socioeconomic groups. Using data from 2003 to 2006, Lostao et al. (2014) suggested that the frequent use of public health care was more common among those with low socioeconomic status but use of private care was more common in higher socioeconomic groups.

In Saudi Arabia, very few studies establish a framework through which to identify the effect of socioeconomic factors on the spatial distribution of households' health status. Abdulelah and Mobaraki (2010) examine the relationship between gender inequity in Saudi Arabia and its relation to public health. They recommend further research to assess knowledge, attitudes, and practices towards health care of Saudi men and women. Asharaf et al. (2014) used analysis of census results to understand regional variations in population and households' condition in Saudi Arabia. Rehmani et al. (2012) conducted a community health survey to describe the health status of National Guard military employees and their dependants residing in the Eastern region of Saudi Arabia. They identify from this survey a baseline from which to strengthen many public health policies and programmes.

More recently, Almubark et al. (2019) describe the distribution of low literacy in the population in Saudi Arabia. More specifically, the authors used a cross-sectional national survey to examine factors associated with health literacy. Both descriptive and a multivariable logistic regression model were developed to found that approximately half of Saudi Arabia has low literacy, and risk factors for low HL were older ages, lower-income and education, having been formerly married, and a moderate pattern of health use.

From the literature, the previous concentrate on determining the nature association of healthcare system with socioeconomic factors. Most of them were conducted in a non-spatial analysis and have not been succeeding to effectively explain the distribution of spatial factors in the healthcare system. This particular study is different from that presented previously regarding the additional advantage given to address the issue. This study is a first and the only one, to our knowledge, that provides spatial statistics that help identify the effect of socioeconomic factors on household's health status in Saudi Arabia.

Our paper has the advantage of utilising explicative analysis rather than purely descriptive, which means that the determinates or factors linked to, or associated with, the household's health status were not well examined. While there is a wealth of research on the healthcare system in Saudi Arabia, studies specifically looking at socioeconomic factors effects on household's health status are limited. The kingdom still lacks more accurate studies on which to identify socioeconomic factors effect and household's health status assessment, and eventually to base management decisions.

The remainder of this paper proceeds in the following way. Section 2 describes the data collected as well as the variables asked at households' level and used in the analysis. The methodology is detailed in section 3 of this paper. Statistical results and analyses are presented in section 4. Conclusion and comments are presented in section 5.

\section{Data Description and Variables}

\subsection{Study Area}

The analysis is at the country level and the geographical focus on these 13 administrative regions (Note 1). The population of the study area is 32.552,336 persons (both Saudis and non-Saudis persons), of whom 24,192,780 (74.32\%) are persons more than fifteen years of age (data available from the General Authority for Statistics). The Household Health Survey Bulletin covers data related to households for all 13 administrative regions of the Kingdom of Saudi Arabia, including: Riyadh, Makkah, Madinah, Qassim, Eastern Region, Aseer, Tabouk, Hail, Northern Borders, Jazan, Najran, Al-Baha, and Al-Jouf (cf. Figure 1).

\subsection{HHS Data}

In 2018, the General Authority for Statistics carried out a survey on Saudi and non-Saudi population, spread out across all administrative regions in the Kingdom of Saudi Arabia, to assess the health status of population. In this survey, the number of Saudi and non-Saudi households claiming poor health status data (HHS) is collected by visiting a representative sample of households listed in the survey sample and accessed them through coordinates on the tablets, guiding maps. A direct contact with the households was adopted as a method to fill the survey questionnaire and collect data. The electronic questionnaire was then completed orally through direct contact with the head of the household or any adult member of the household who is familiar with its affairs. The questionnaire contained a series of questions that meet the objective of the study. The majority of the questions were designed in 
such a way that households expressed their health status. Only persons (15 years and above) who assessed their own health are considered in the analysis. For the empirical analysis, the HHS is measured by the percentage of Saudi and non-Saudi individuals who assessed their own health as non-good by administrative region.

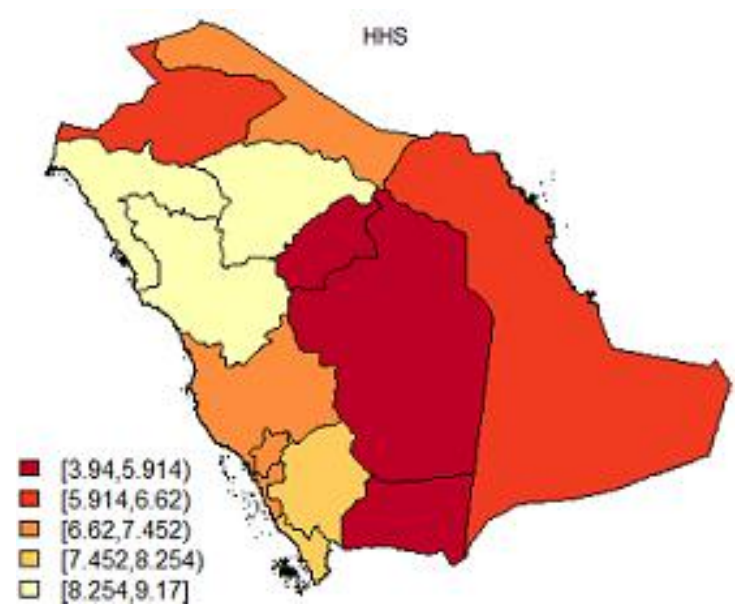

Figure 1. Map of the household's health status distribution in Saudi Arabia (thirteen administrative areas)

Figure 1 shows a marked variation in the number of Saudi and non-Saudi households claiming poor health status between different administrative regions, ranging between 3.94 and 9.17 percent. Since the identification of Saudi and non-Saudi health status is difficult at any time, the major preventive action available is to understand the geographic trends of HHS and delimit administrative regions where individuals suffering from non-good health status. Thus, identification of socioeconomic factors impacting the Saudi and non-Saudi health status could provide targets for preventive action as adjusting control measures.

\subsection{Variables}

A large number of factors, many of which are beyond the formal authority of the health care system, affect Saudi and non-Saudi health status. At the conceptual level, determinants described the way in which many factors combine to affect the health of individuals, and they explained that, to a large extent, such aspects as where an individual lives, and the state of her/his environment, genetics, income, education, and relationships with friends and family all have considerable impacts on health.

For our case, the number of Saudi and non-Saudi households claiming poor health status (HHS) findings are explored and discussed according to likely contributing factors. Among the various factors which affect the health status of Saudi and non-Saudi households, particular importance should be attached to average monthly income (AMI), seeing that in present world condition other factors, such as the Saudi expenditure health (SEH), Saudi unemployment rate (SUR), Percentage of households access to health services (DHF) measured by the distance of residence from health facilities and administrative area, Number recreation \& Culture/100 Beads (NRC), Saudi population covered by insurance (PCHI), and current daily smokers (CDS). Data related to these factors were obtained from the General Authority for Statistics in Saudi Arabia. These data were used to identify the relationship between socioeconomic factors and the number of Saudi and non-Saudi households claiming poor health status. The analysis allowed the establishment of disadvantaged areas for each Saudi population health is identified as poor. 


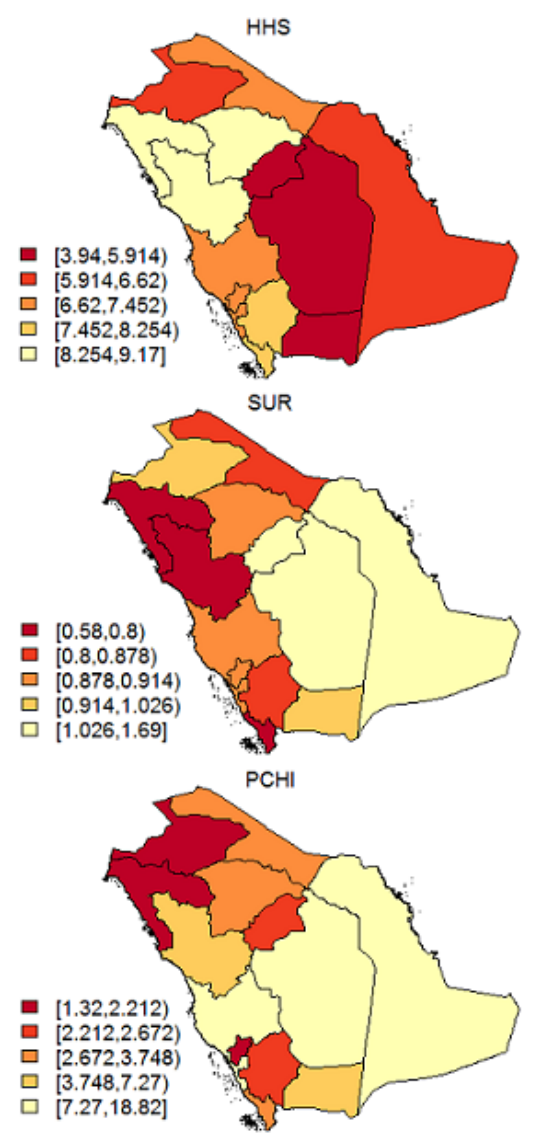

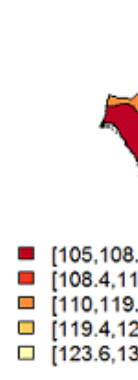
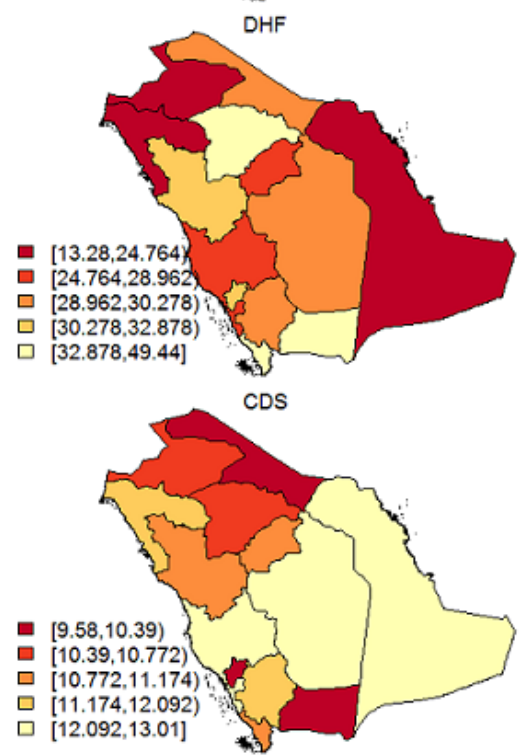

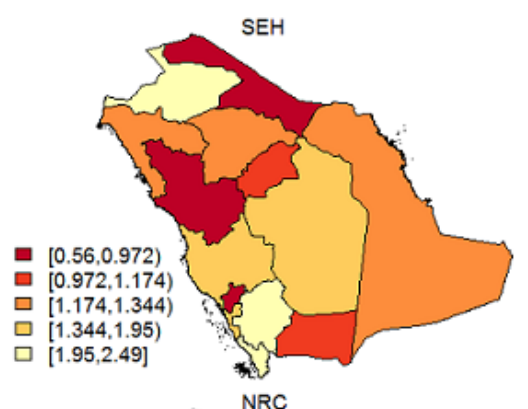

$\mathrm{NRC}$

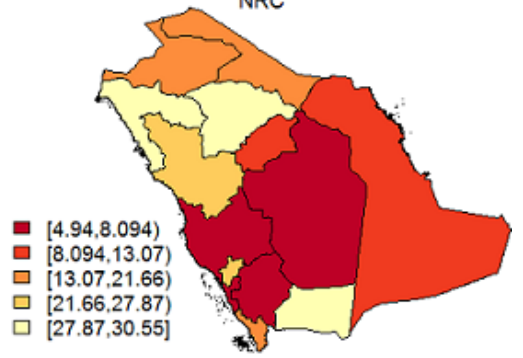

Figure 2. Map of the distribution of Saudi and non-Saudi household's health status (HHS) and the socioeconomic factors retained in the analysis

Figure 2 shows that HHS and socioeconomic factors retained in this paper have approximately the same spatial patterns. Therefore, it is clear that an $a$ priori association between HHS and socioeconomic factors is one for empirical investigation. With this intention, the used data were entered and validated using internal consistency checks and analysed in statistical system $\mathrm{R}$ version 3.1.0 (available at http://cran.r-project.org).

\section{Methods}

The methodology of this study was primarily qualitative. The data was collected through a questionnaire containing multiple questions regarding the health status of members of Saudi society. We note that the target statistical population of the Household Health Survey is composed of all individuals (Saudis and non-Saudis) who reside in Saudi Arabia. The survey sample was chosen by identifying 24,012 households as a selected sample that represents the survey population at the level of kingdom and is distributed among the thirteen administrative regions in Saudi Arabia. Data related to the Household Health Survey is displayed in suitable tables to facilitate summarizing, understanding, and drawing analyses from them.

A Generalized Linear Spatial Model (GLSM) is used to model the spatial dependence between the numbers of households recorded in the Saudi districts. The number of households in district $i$ is the response variable assumed

to be Poisson. Parameters of this Poisson GLSM are $\theta=\left(\sigma^{2}, \phi\right)$ and

$\beta=\left(\beta_{0}, \beta_{1}, \beta_{2}, \beta_{3}, \beta_{4}, \beta_{5}, \beta_{6}, \beta_{7}\right)$, where $\beta_{0}$ the intercept and $\beta_{1}, \beta_{2}, \beta_{3}, \beta_{4}, \beta_{5}, \beta_{6}, \beta_{7}$ are the regression coefficients corresponding to the average monthly income (AMI), the Saudi expenditure health (SEH), the Saudi unemployment rate (SUR), the percentage of households access to health services, measured by the distance of 
residence from health facilities and administrative area (DHF), Number recreation \& Culture/100 Beads (NRC), the Saudi population covered by insurance (PCHI), and the current daily smokers (CDS) respectively. Conditionally on the Gaussian process $S$ with mean 0 and variance $\sigma^{2}$, the response variable $H H S_{i}$, for $i=1, \ldots, 13$, are modeled as independent Poisson variables with mean $\lambda_{i}$, where

$$
\begin{gathered}
\ln \left(\lambda_{i}\right)=\beta_{0}+\sum_{i=1}^{7} \beta_{j} x_{i j}+S_{i} \\
\ln \left(\lambda_{i}\right)=\beta_{0}+\beta_{1} A M I+\beta_{2} S E H+\beta_{3} S U R+\beta_{4} D H F+\beta_{5} N R C+\beta_{6} P C H I+\beta_{7} C D S+S_{i}
\end{gathered}
$$

This spatial Poisson regression is based on statistical methods and geoRglm software package (http://cran.r-project.org) developed by Diggle et al., (Diggle et al., 2002) for the generalized linear spatial models. In this regression, HHS for the districts was regressed on the socioeconomic factors for the thirteen studied regions.

A $\log$-link function, a powered-exponential spatial auto-correlation function $\rho(h)=\exp (-h / \phi)$ for the Gaussian

process for $S$, and a Bayesian inference framework were used (Diggle et al. (1998; 2002)).

\section{Results}

According to the result of the population census of 2018 carried by the General Authority for Statistics, Saudi Arabia has a population of 32,552,336. Most of them (56.7\%) are Saudi natives and the rest $(43.3 \%)$ are non-Saudi immigrants from various parts of the world (Tale 1). Immigrants to Saudi Arabia come from many different countries and culture and mostly from Southeast Asia and Africa (Khraif; 2009). While (79.33\%) of the Saudi natives live in less urbanized regions such as Tabouk (79.41\%), Northern Borders (79.11\%), Al-Baha (80.32\%), and Asser (79.12\%), non-Saudi population is concentrated in highly urbanized regions such as Al-Riyadh (43.7\%), Makkah Al-Mokarramah (47.22), Al-Madinah Al-Monawarah (35.47), and Eastern Region (35.92), indicating a structural change in labor force, shifted from agriculture to the sector service.

Table 1. Descriptive statistics of spatial distribution of Saudi population by administrative region

\begin{tabular}{lcccccc}
\hline Administrative region & $\begin{array}{c}\text { Number of } \\
\text { households }\end{array}$ & $\begin{array}{c}\text { Saudi and } \\
\text { non-Saudi }\end{array}$ & $\begin{array}{c}\text { Percent } \\
\text { Saudi }\end{array}$ & $\begin{array}{c}\text { Region } \\
\text { wise }(\%)\end{array}$ & $\begin{array}{c}\geq 15 \text { years } \\
(\%)\end{array}$ & $\begin{array}{c}\text { HHS } \\
(\%)\end{array}$ \\
\hline Al-Riyadh & 3726 & 8216284 & 56.70 & 25.24 & 22.48 & 25.31 \\
Makkah Al-Mokarramah & 4356 & 8557766 & 52.78 & 26.29 & 22.99 & 24.42 \\
Al-Madinah Al-Monawarah & 1656 & 2132679 & 64.53 & 6.55 & 6.58 & 29.59 \\
Al-Qaseem & 1188 & 1423935 & 70.90 & 4.37 & 4.92 & 25.32 \\
Eastern Region & 3024 & 4900325 & 64.08 & 15.05 & 15.33 & 25.24 \\
Aseer & 1620 & 2211875 & 79.12 & 6.79 & 8.54 & 29.17 \\
Tabouk & 1314 & 910030 & 79.41 & 2.80 & 3.40 & 21.33 \\
Hail & 1170 & 699774 & 76.90 & 2.15 & 2.65 & 28.46 \\
Northern Borders & 1260 & 365231 & 79.11 & 1.12 & 1.37 & 30.14 \\
Jazan & 1278 & 1567547 & 77.02 & 4.82 & 6.03 & 30.06 \\
Najran & 1152 & 582243 & 75.23 & 1.79 & 2.04 & 29.02 \\
Al-Baha & 1080 & 476172 & 80.32 & 1.46 & 1.98 & 27.35 \\
Al-Jouf & 1188 & 508475 & 74.68 & 1.56 & 1.69 & 30.62 \\
\hline Total & 24012 & 32552336 & 62.69 & 100.00 & 100.00 & - \\
\hline
\end{tabular}

On the other hand, the Makkah Al-Mokarramah concentrates the largest share of the population (26.29\%) followed by Al-Riyadh (25.24\%), and Eastern Region (15.05\%); thus totaling $66.58 \%$. This imbalance of population may have 
been due to cultural and economic factors. These three regions are highly developed and constitute metropolises with a high level of industrial and commercial activity (Khraif; 2000). Since these metropolises attract both non-Saudi manpower from other countries and also Saudi manpower from other regions, they have created an imbalance of population in Northern Borders, Al-Jouf, Al-Baha, and Najran regions (Khraif 1994).

The socioeconomic factors used in this analysis are likely to affect the health status of individuals in Saudi Arabia, but the underlying variables - unemployment, smoking, low income, and others - tend to be under-examined and under-studied. Moreover, the number of Saudi and non-Saudi individuals (15 years and above) who member assessed their own health status (good or very good) by administrative region is small relative to the population suffering from poor health status (HHS (\%) is relatively high in Table 1). Thus, a better understanding of household's health status determinants is certainly desirable in its own right, for policymakers with the specific goal of improving Saudi and non-Saudi health status. With these policy implications in mind, the current paper examines the link between Saudi and non-Saudi health status and socioeconomic factors, a long-standing empirical question in Saudi social and health research.

Table 2. Estimates and 95\% intervals for the parameters of the spatial Poisson regression model

\begin{tabular}{lcccc}
\hline Parameter & Posterior mean & $2.5 \%$ quantile & $97.5 \%$ quantile & Posterior median \\
\hline$\beta_{0}$ (Intercept) & -1.701 & -5.015 & 4.034 & -1.823 \\
$\beta_{1}$ (AMI) & $-0.825^{*}$ & -1.673 & -0.042 & -0.795 \\
$\beta_{2}$ (SHE) & -0.131 & -2.966 & 1.624 & -0.126 \\
$\beta_{3}$ (SUR) & $1.309^{*}$ & 0.164 & 5.606 & 1.401 \\
$\beta_{4}$ (DHF) & 1.873 & -0.109 & 3.425 & 1.765 \\
$\beta_{5}$ (NRC) & -0.332 & -1.161 & 0.887 & -0.334 \\
$\beta_{6}$ (PCHI) & -0.020 & -0.078 & 0.054 & -0.021 \\
$\beta_{7}$ (CDS) & $0.023^{*}$ & 0.001 & 0.083 & 0.019 \\
$\phi$ & 112.565 & 80.236 & 165.666 & 111.555 \\
$\sigma^{2}$ & 0.897 & 0.236 & 1.946 & 0.947 \\
\hline
\end{tabular}

"Indicates parameters that are significant at 5\%

At administrative regions level, Table 2 shows parameter estimates from the Poisson spatial regression. The results obtained from the spatial analysis indicate a significant negative relationship between HHS and the average monthly income of the household: what this means that the percentage of Saudi and non-Saudi who do not have good health status decreases with increasing average monthly income (coef $=-0.825,95 \%$ confidence interval: $(-1.673,-0.042)$ ). This finding is in line with the findings of a pilot study on the impact of socioeconomic factors on child oral health-related quality of life (COHRQoL) conducted by Piovesan et al., (2010) in Brazil. They found that higher impacts on COHRQoL were observed for children with lower household income. 
Our second result suggests that the unemployment rate is a significant risk factor for household health status in Saudi Arabia. Table 2 shows that the percentage of Saudi and non-Saudi who do not have good health status increases with the increasing unemployment rate (coef. $=1.309,95 \%$ confidence interval: $(0.164,5.606)$ ). Thus, long-term unemployment is a more important determinant of HHS, a result that is particularly strong for policymakers in Saudi Arabia. This perspective joins that of Timothy et al., (2012) who have suggested that there is a positive association between unemployment and suicide in US states from 1996 to 2005. This is also supported by Donyavi et al., (2011) that in Iran the unemployed and retired individuals showed a higher risk of mortality after myocardial infarction. Consistent with this, unemployment was one of the most significant contributing socioeconomic factors to have negative effects on HHS in Saudi Arabia.

The third, and last, findings concern the statistical association between individuals' smokers and their health status. On this basis, it is apparent from Table 2 that the percentage of individuals smokers increased significantly with the percentage of Saudi and non-Saudi who do not have good health status (coef. $=0.023,95 \%$ confidence interval: 0.001, 0.083). A similar result is justified in a case such as this, in which Nato et al., (2005) examine the association between tobacco smoking, in particular water pipe smoking, and periodontal health. They provided convincing evidence to support the role of tobacco smoking as a risk factor for periodontal disease. The authors showed that both water pipe and cigarette smokers exhibited a greater mean probing depth and a higher prevalence of the disease than non-smokers, suggesting that both types of tobacco smoking exerted a negative influence on periodontal health.

As a result, we carefully conclude that household income, unemployment, and household smoking are the main factors affecting the health status of Saudi and non-Saudi population. Thus, this particular strong information will help to further raise public awareness of the importance of good-health determinants. Consequently, the Saudi government efforts will be oriented toward the objectives aiming to improve awareness in the public about smoking danger. More details are provided on this issue in the following section.

\section{Discussion and Conclusion}

This paper is a first attempt in addressing the household's health status in Saudi Arabia. It is intended to provide a situational analysis of the health of both Saudi and non-Saudi households. Compounding the motivation for addressing this topic is essentially the lack of data and research about the household's health status in Saudi Arabia. Besides, this work was designed to a better understanding of how socioeconomic factors can affect the general situation of the health status of the Saudi population. First, by neglecting the time dimension, we attempted to estimate the HHS in the whole kingdom during 2018. Second, using spatial Poisson models, we modelled HHS as a function of socio-economic factors in the different geographic administrative districts. As noted earlier, the numerous works, previously introduced, are not statistically comparable with our study for three reasons: firstly they use non-Bayesian approach; secondly, their regression parameters are marginally interpreted whereas ours are to be interpreted conditionally on the unobserved Gaussian process and thirdly their analysis is carried out in accordance with standard methods and using classical software programme whereas ours are developed in spatial analysis context.

By regressing the number of Saudi and non-Saudi households claiming poor health status (HHS) in the whole kingdom during 2018 on socio-economic factors, it was verified that average monthly income (AMI) is significantly and negatively correlated $(<0.05)$ with HHS, meaning that proportion of households claiming poor health status decreases with their average monthly income. This view is strongly supported by the economic literature, which suggested that income is an important factor that significantly affects an individual's health status (Zhao, 2007a). Furthermore, the statistical results reported in our study prove that the number of Saudi and non-Saudi households claiming poor health status is significantly and positively correlated with the Saudi unemployment rate (SUR) on one hand and current daily smokers (CDS) on the other hand. This result is somewhat surprising because, as Healy (2003) noted that poor health status is not generally well correlated with low socio-economic factors, again a finding not consistent with the idea that poverty due to unemployment, for example, is the main cause which can disturb the equilibrium of human health.

In terms of the policy, the health of the population is defined as a special social interest in Saudi Arabia, in the light of the government-financed programs in the field of health that will benefit the Saudi community. However, these programmes and policies are probably misguided since the higher number of Saudi and non-Saudi households claiming poor health status (cf. HHS in Table 1). The situation would be further aggravated if the number of individuals suffering from bad health status in the Saudi community in the coming years will be high. To avoid this situation, decision-making authority interventions directed toward smoking and non-smoking, say through an awareness system would be worthwhile. However, the set of policy interventions to improve population health will 
vary depending upon which aspects of socioeconomic determinants are responsible for increased the number of Saudis suffering from bad health status.

The association between HHS and the Saudi unemployment rate (SUR) is much stronger than the relationship between HHS and the average monthly income in absolute value in the one hand, and the association between HHS and smoking in the other hand in the other hand. This may simply reflect that as Saudi households are unemployed the psychological cost tends to increase and consequently household resources (income) tend to decline. In this case, one generally might expect a bad effect on the health status of individuals since the underlying pathologies depression, smoking, other mental illness - tend to appear causing bad health status in the Saudi population.

The analysis carried out in this paper seeks to demonstrate the role of socioeconomic factors in determining the Saudi and non-Saudi household's health status. In this respect, there is very little empirical research regarding the causal link between the household's health status and socioeconomic conditions undertaken previously. Most of these researches are fundamentally biased and can lead to misleading conclusions about socioeconomic impacts associated with current and future Saudis and non-Saudis health status. An important aspect of this paper is providing information to policymakers for both integrating socioeconomic effects on household's health status and deciding where to make resources and aid available, and ultimately improve Saudi and non-Saudi population health status. Current research focuses on the analysis of socioeconomic determinants of Saudi household's health status using GLSM. Future work will concern the Spatio-temporal modelling (Picado et al. 2007) of HHS to see if there has been a fairly substantial improvement in the Saudi household's health status.

Finally, the main socioeconomic determinants of Saudi and non-Saudi household's health status must be understood by the government with the specific goal of lowering the number of individuals suffering from bad health status. While better strategies, decisions, and health programs are certainly desirable, for policy-makers, to achieve better population health, interventions broadly directed toward these strategies and programs may be less cost-effective than focusing more narrowly on factors that cause bad health for individuals, such as unemployment, lower average monthly income, and currently daily smoking. The Saudi government is expected to concentrate on providing awareness, say about the danger of smoking since people are rational and are responsive to relevant information. Moreover, Saudi authority efforts will be more oriented towards developing strategies and health programs taking into account that lower-income and unemployment affect significantly the Saudi household's health status.

\section{References}

Abdulelah M., \& Söderfeldt, B. (2010). Gender inequity in Saudi Arabia and its role in public health. Eastern Mediterranean Health Journal, 16(1), 113-118.

Almubark, R., Basyouni, M., Alghanem, A., Althumairi, N., Alkhamis, D., Alharbi, L. S., ... BinDhim N, (2019). Health literacy in Saudi Arabia: Implications for public health and healthcare access. Pharmacology Research \& Perspectives, 7(4), 1-10.

Asharaf, A. S., Ibrahim, E., Rshood, K., \& Abdullah, A. (2014). Population distribution and household conditions in Saudi Arabia: reflections from the 2010 Census. Springer Plus, 3(530), 1-18.

Bago d'Uva, T., Jones, A. M., \& Van, D. E. (2009). Measurement of horizontal inequity in health care utilisation using European panel data. Journal Health Econ., 28, 280-289.

Devaux, M., \& De Looper, M. (2012). Income-Related Inequalities in Health Service Utilization in 19 OECD Countries, OECD Health. Working Papers, No. 58. Paris: OECD Publishing.

Diggle, P. J., Tawn, J., \& Moyeed, R. (1998). Model-based geostatistics. Appl. Stat., 47, 299-350.

Diggle, P., Moyeed, R., Rowlingson, B., \& Thomson, M. (2002). Childhood malaria in the Gambia: a case-study in model-based geostatistics. Appl. Statist., 51, 493-506.

Donyavi, T., Naieni, K. H., Nedjat, S., Vahdaninia, M., Najafi, M., \& Montazeri, A. (2011). Socioeconomic status and mortality after acute myocardial infarction: a study from Iran. International Journal for Equity in Health, $10(9), 1-10$

Healy, J. D. (2003). Excess winter mortality in Europe: a cross country analysis identifying key risk factors. Journal of Epidemiol Community Health, 57(10), 784-9.

Hilary, D., Sue, S., Bornstein, M. D., Gregory, C., \& Kane, M. D. (2018). Addressing Social Determinants to Improve Patient Care and Promote Health Equity: An American College of Physicians Position Paper. Annals of Internal Medicine, 168(8), 577-578. 
Jenni, B., \& Lauri, J. V. (2020). Socioeconomic differences in use of public, occupational, and private health care: A register-linkage study of a working-age population in Finland. PLOS ONE, 15(4), 1-18.

Khraif, R. M. (1994). Residential Mobility in the City of Riyadh: a Study of its Directions, Reasons and Characteristics. Occasional Paper Published by the Saudi Geographical Society. King Saud University, Riyadh.

Khraif, R. M. (2000). The Labor Force in Saudi Arabia: Spatial Dimensions and Socioeconomic and Demographic Characteristics. Occasional Papers Published by Saudi Geographical Society. King Saud University, Riyadh.

Khraif, R. M. (2009). Fertility Behaviors of Grazing Groups in Suman and Northern Parts of Saudi Arabia. Paper Presented at XXVI IUSSP International Population Conference, Marrakesh Morocco 27 Sep-2 Oct.

Lostao, L., Blane, D., Gimeno, D., Netuveli, G., \& Regidor, E. (2014). Socioeconomic patterns in use of private and public health services in Spain and Britain: implications for equity in health care. Health Place, 25, 19-25.

Natto, S., Baljoon, M., \& Bergstrom J. (2005). Tobacco Smoking and Periodontal Health in a Saudi Arabian Population. Tobacco Smoking and Periodontal Health, 76(11), 1919-1926.

Picado, A., Guitian, F. J., \& Pfeiffer, D. U. (2007). Space-time interaction as an indicator of local spread during the 2001 FMD outbreak in the UK. Preventive Veterinary Med., 79, 3-19.

Piovesan, C., Antunes, J. L. F., Guedes, R. S., \& Ardenghi, T. M. (2010). Impact of socioeconomic and clinical factors on child oral health-related quality of life (COHRQoL). Quality Life Research, 19, 1359-1366.

Regidor, E., Martınez, D., Calle, M. E., Astasio, P., Ortega, P., \& Domınguez, V. (2008). Socioeconomic patterns in the use of public and private health services and equity in health care. BMC Health Service Research, 8, 1-9.

Rehmani, R., Elzubair, A. G., Al Maani, M., Chaudary, I. Y., Al Qarni, A., Khasshogi, T., \& Al Shuaibi, A. (2012). Population-based health survey in Eastern region of Saudi Arabia. Eastern Mediterranean Health Journal, 19(5), 417-425.

Roos, N. P., \& Mustard, C. A. (1997). Variation in health and health care use by socioeconomic status in Winnipeg, Canada: does the system work well? Yes and no. Milbank $Q, 75,89-111$.

Timothy, J. C., \& Richard, A. D. (2012). The effect of job loss and unemployment duration on suicide risk in the united states: a new look using mass-layoffs and unemployment duration. Health Economics, 21, 338-350.

Van, D. E., Masseria, C., \& Koolman, X. (2006). Inequalities in access to medical care by income in developed countries. CMAJ, 174, 177-183.

Zhao, J. (2007a). Socio-Economic Determinants of Health and Health Care Utilization of Recent Immigrants in Canada. Prepared for Citizenship and Immigration Canada.

\section{Note}

Note 1. An administrative region is a part of Saudi Arabia that is run by a governmental agency affiliated to the Ministry of the Interior. 